\title{
Guest Editorial: Nanophotonics for Space Applications
}

\author{
Edward W. Taylor \\ International Photonics Consultants \\ 38 Knife Edge Place, Pagosa Springs, Colorado 81147, USA \\ IntPhoton@aol.com
}

The application of cutting-edge photonics technology for improving the performance of space-based assets has resulted in a myriad of success stories. As early as 1981, the first photonics-based active fiber optic data links aboard the NASA Long Duration Exposure Facility (Air Force Experiment, AFWL Experiment 701, Fiber Optic Space Effects), during a 69-month orbital period in the Van Allen radiation belts convincingly demonstrated that optical fibers, III-IV laser diodes and detectors, and other photonics technology to follow would have a major role in reducing the dependency on expensive, complexly-wired system technologies, while reducing weight and volume. The proof-of-concept experiment verified the contention that reduced-size photonic systems could reduce satellite weight and volumes while dramatically increasing the bandwidth, reliability, and survivability of commercial and military satellite communication and data acquisition systems. Most importantly, the experiment provided the first conclusive evidence that properly designed photonic systems could provide excellent resistance to the natural space radiation and temperature environment.

More recently, QD and nanostructure-based photovoltaic and nonlinear components and sensor arrays operating from visible wavelengths to far-IR are under investigation for near-term application in space systems. Cutting-edge photonic-nanophotonic components, materials, and systems under investigation include radiation-resistant, QD-based chip-sized nonlinear inertial guidance systems, focal plane arrays, thin film solar cells, polymer detectors, polymer modulators, active-smart optical windows, and specialized coatings, to name but a few applications, several of which are presented in this special section.

The recent evolution of exotic photonic technology involving integration of nanotechnology with polymers and new molecular-hybrid nanomaterials have shown a high potential for potentially improving specific extra-terrestrial applications, as discussed in this special section. The diverse potential applications are well represented in this section and include papers on protective optical limiting coatings for spacebased sensors (Sarkar et al.), radiation-resistant properties of photovoltaic solar cells (Hubbard et al.), mitigation of Lunar regolith using nanoparticle smart coatings (Pirich et al.), and evolving radiationresistant EO polymer modulator materials (Zhang and Taylor).

Recently announced plans by NASA to return to the Moon in 2020 as well as other potential interplanetary space missions will require lightweight polymer-based shielding materials for crew cabins and the protection of astronauts and components from galactic cosmic rays (GCR) during lunar surface exploration and interplanetary missions. Nanotechnology is expected to play a role in the development of some of these materials. Traditional radiation high- $Z$ shielding materials such as lead and other heavy elements are not suitable for GCR protection since the interaction of GCR with these materials results in the production of secondary radiation which is harmful to humans and can degrade photonic and electronic components. Radiation transport calculations have shown that hydrogen-rich low- $Z$ materials are more effective per unit mass of shield than heavier shielding elements for $\mathrm{x}$ - or gamma-ray absorption because of their lower cross sections, and because heavier elements can serve as the sources of dose-producing secondary particles such as short-range heavy nuclear fragments and penetrating neutrons. Thus, a challenge to develop new nano-based materials and radiation-resistant photonic components for space applications appears to be well under way.

Hopefully the initial sampling of papers presented in this special section on Nanophotonics for Space Applications will be well received and provide new ideas from the readership for addressing the special needs of the photonics space community. 